www.nature.com/pj

\title{
Laminated film composites of multilayered plastic film and inorganic polymer binder as an alternative to transparent and hard glass
}

\author{
Polymer Journal (2013) 45, 685-689; doi:10.1038/pj.2013.28; published online 27 March 2013
}

\begin{abstract}
INTRODUCTION
Various types of glasses, soda-lime, Pyrex, quartz, and crown glass are used as hard and transparent materials for a variety of industrial applications such as windows, displays, screens and lenses. ${ }^{1}$ However, glass materials have several limitations. First, glass is quite heavy and is two to three times heavier than plastic. $^{2,3}$ Second, glass is brittle with low toughness, which readily leads to catastrophic cracks and broken pieces; this can cause mechanical damage that injures humans. ${ }^{4-6}$ To reduce the brittleness and to increase the strength, glass surfaces are processed by controlled thermal and chemical treatments. For example, Gorilla Glass ${ }^{7}$ is chemically strengthened through an ion-exchange $\mathrm{e}^{8,9}$ process that creates a deep compression layer on the surface of the glass substrate. These glasses have high compressive stress and are widely used as protective covers for electronic displays such as laptop computer screens and mobile devices. However, the sophisticated processes involved are expensive, and the large weight is still problematic. $^{10}$
\end{abstract}

Plastics are being considered as a light and transparent alternative material to heavy and brittle glass. In particular, plastic substrates are of great interest for applications in flat panel displays and optical industries. Poly (ethylene terephthalate) (PET), poly(methyl methacrylate), polycarbonate and polyethersulfone have been used to replace heavy and fragile glass substrates. ${ }^{11}$ For example, PET has lower light loss and greater transmittance at visible wavelengths than normal glass and thus has merit as a transparent substrate. ${ }^{12}$ It has been applied to various display and optical products such as windows of mobile phones, car navigation systems and digital multimedia broadcasting (Figures 1a and b). However, despite these advantages, a low Young's modulus and low surface hardness limit its applicability. Various attempts have been made to improve the modulus of bulk plastic materials and surface softness by using organic-inorganic hybrid composites and coating techniques. ${ }^{13}$ Recently, polyhedral oligomeric silsesquioxane (POSS) nanocages have attracted attention owing to their observed mechanical and physical enhancement of a variety of organic systems as an inorganic polymer reinforcement (Figure 1c). ${ }^{14,15}$ In particular, the incorporation of a reactive group containing POSS facilitates consolidation through simple ultraviolet (UV) or thermal exposure in the presence of suitable additives. ${ }^{16}$ Rather than the dispersed composite phase of binary organic and inorganic components with different homogeneities as previously reported, the laminating approach is an interesting and fresh concept that may be able to achieve lightweight glass-like materials, while multilayered plastic film composites with high flexibility have been used for packaging applications such as food packing, tubing and boxes, as well as sandwiching glass plates with plastic film for safety glass applications. ${ }^{17}$

In this paper, we propose a new approach to fabricating laminated film composites with a high modulus, transparency and lightweight as an alternative to glass. The laminated film composite was fabricated by alternately layering plastic PET films and methacrylate-POSS inorganic polymer interlayers and a subsequent simple UV curing process. The UV-curable viscous inorganic polymer methacrylate-POSS was mixed with an organic additive to strongly bind the PET film layers together through the cross-linking reaction. The obtained multilayered PET/ POSS composites were evaluated to determine the modulus, transparency and haze.

\section{MATERIALS AND METHODS}

\section{Materials}

Commercially available $100 \mu \mathrm{m}$ thick malinex PET film (Teijin DuPont Films, Tokyo, Japan) was cut into pieces. 3-(3,5,7,9,11,13,15-Heptacyclopentylpentacyclo[9.5.1.1.1.1]-octasiloxane-1-yl) propyl methacrylate was purchased from Hybrid Plastics Co. (Hattiesburg, MS, USA). Ethylene glycol dimethacrylate (EGDMA) monomer, 2,2'dimethoxy-2-phenylacetophenone, 2,4,6,8-Tetramethyl-2,4,6,8-tetravinylcyclotetrasiloxane (V4D4), tertbutylperoxide (TBPO) and trichlorovinylsilane (TVC primer) were all purchased from Sigma Aldrich Chemical Co. (Milwaukee, WI, USA).

The surface modification of PET film by initiated chemical vapor deposition process The surface of PET film was modified by three steps to enhance the adhesion with inorganic binder. At first, an oxygen plasma (Daeki High Tech., Daejeon, Korea) was treated on the PET film surface for $1 \mathrm{~min}$ to create reactive hydroxyl groups. Second, to introduce vinyl groups on the surface of PET film, TVC primer as a coupling agent was spin coated on surface at 3000 r.p.m. for 30 s. Finally, the monomer with vinyl groups was coated by initiated chemical vapor deposition (iCVD) process described in detail at elsewhere. ${ }^{18}$ In typical, V4D4 monomer was charged into a source cylinder and heated to $70^{\circ} \mathrm{C}, 1 \mathrm{ml}$ of TBPO was also added into a source cylinder on heating. Then both the monomer and initiator were vaporized and introduced into the reactor at the flow rate ratio of $\mathrm{V} 4 \mathrm{D} 4: \mathrm{TBPO}=2: 1$. The polymerization reaction was initiated with the filament at $200^{\circ} \mathrm{C}$. The temperature of PET substrate was maintained at $40^{\circ} \mathrm{C}$. The process 

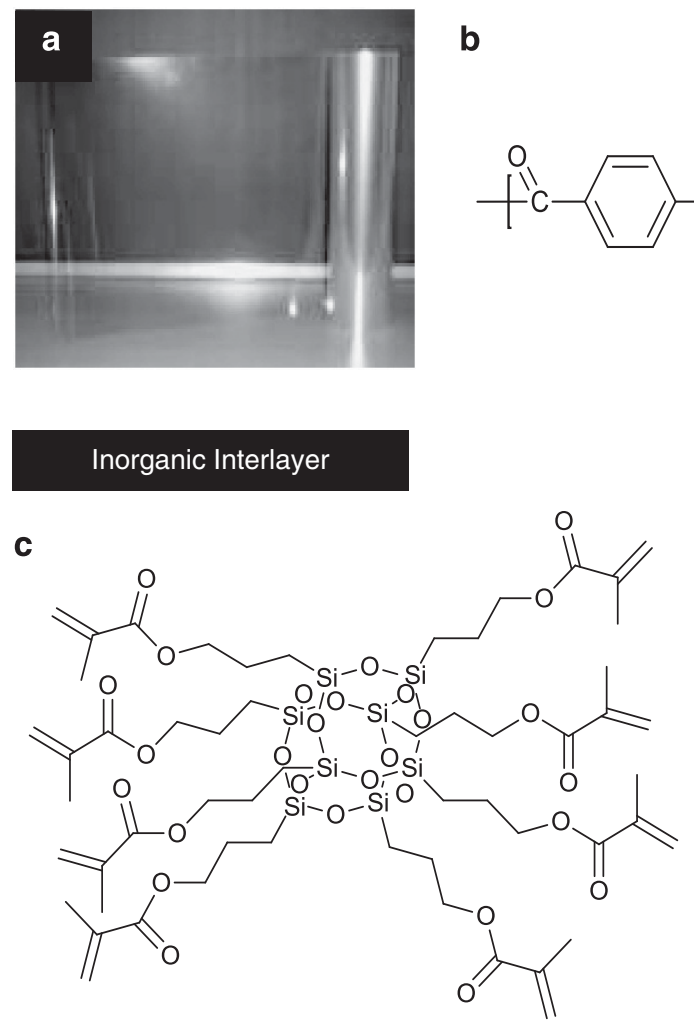

d<smiles>C=C(C)C(=O)OCCOC(=O)C(=C)C</smiles>

Figure 1 Components for fabrication of the laminated film composites. (a) The PET plastic film and (b) its chemical structure. Chemical structure of (c) POSS inorganic polymer interlayer and (d) EGDMA additive. A full color version of this figure is available at Polymer Journal online.

pressure was $100 \mathrm{~m}$ Torr. The deposition was monitored in situ by interferometry for $20 \mathrm{~min}$.

\section{Fabrication of laminated film composite}

The fabrication procedure for the proposed laminated film composite is specified below in detail. (1) A PET film modified by the iCVD method was cut to make each type of laminated composite sample. POSS/EGDMA (inorganic polymer/ crosslinker $=1: 1$ weight) mixed with $0.2 \mathrm{wt} \%$ 2,2'-dimethoxy-2-phenylacetophenone photoinitiator was homogeneously coated onto the modified PET film at several microliters per square micrometer for a thickness of $\sim 5 \mu \mathrm{m}$. (2) The POSS polymer and PET film were alternately stacked to produce a 11-layer (6 PET films +5 POSS interlayers) composite, which was then subjected to UV exposure (ELC-4100 UV light system) for $20 \mathrm{~min}$. The inorganic polymer interlayer binder was solidified to produce transparent and hard composite samples with strong adhesion between the PET films (Figure 2). To measure the mechanical and physical properties, laminated film composites with two different sizes were prepared: $0.3 \mathrm{~cm}$ (width) $\times 3 \mathrm{~cm}$ (length) $\times 0.063$ $\mathrm{mm}$ (depth) for the three-point bending test and $2 \times 2 \mathrm{~cm} \times 0.063 \mathrm{~mm}$ for the transparency and haze tests. In addition, POSS-only and glass samples were prepared with the same dimensions for comparison of the mechanical strength and optical properties.

\section{Measurements of mechanical and physical} properties, and characterization

The flexural strength was measured by a threepoint bending test using a universal testing machine (RB Model 302 ML, R\&B, Deajeon, Korea) with a 2-kgf load cell at a crosshead speed of $0.2 \mathrm{~mm} \mathrm{~min}^{-1}$. This measurement was performed according to ASTM E 855. The tests were done on five different specimens, and the average value was calculated as the final value with a s.d. The transparency and haze values of the laminated film composites were measured using a haze meter NDH 5000 (Nippon Desnshoku Industries Co. Ltd, Tokyo, Japan). The total luminous transmittance of the transparent materials was measured in accordance with JIS K7361-1 using a single beam instrument; the haze of the composite was determined in accordance with JIS K7136.

Cross-sectional images of the laminated film were measured using scanning electron microscopy (SNE-4500 M, SEC, Suwon, Korea) at an accelerating voltage of $15 \mathrm{kV}$ after Pt sputtering for $20 \mathrm{~s}$. The $\mathrm{X}$-ray photoelectron spectroscopy spectra of the modified PET film used in this study were obtained with a MultiLab 2000 spectrometer (Thermo Scientific, Waltham, MA, USA). Al Ka $(1485.6 \mathrm{eV}) \mathrm{X}$-rays were used with $14.9 \mathrm{keV}$ anode voltage, $4.6 \mathrm{~A}$ filament current and $20 \mathrm{~mA}$ emission current. Each sample was treated at $10^{-9}$ mbar to remove impurities. The survey spectra were obtained at $50 \mathrm{eV}$ pass energy and $0.5 \mathrm{eV}$ step size.
Core level spectra were obtained at $20 \mathrm{eV}$ pass energy and $0.05 \mathrm{eV}$ step size. The surface topography was taken using atomic force microscopy (XE-100, Parksystem, Parksystem, Suwon, Korea) recorded with standard tips in noncontact mode at a scan rate of $0.7 \mathrm{~Hz}$.

\section{RESULTS AND DISCUSSION}

POSS is a cage-like silsesquioxane with an inner inorganic silicone $\left(\mathrm{SiO}_{1.5}\right)_{\mathrm{x}}$ framework that is considered to be $1-3 \mathrm{~nm}$ in size, similar to that of a silica particle. ${ }^{19}$ In contrast to conventional inorganic fillers, POSS has low density but useful properties, such as high thermal stability and good oxidation resistance. The controlled functional groups in the organic substituent bring chemical versatility to POSS and make it widely compatible with various polymers. In this work, a methacrylate-functionalized POSS monomer was found to be readily miscible with EGDMA additive with low viscosity ( $\sim 4 \mathrm{cPs}$ ), which reduced the high viscosity of POSS for better processability; the UV curing reaction to consolidate the polymer occurred by free radical polymerization in the presence of a photoinitiator.

The well-defined polymerization chemistry of the iCVD process allows the surface 
a

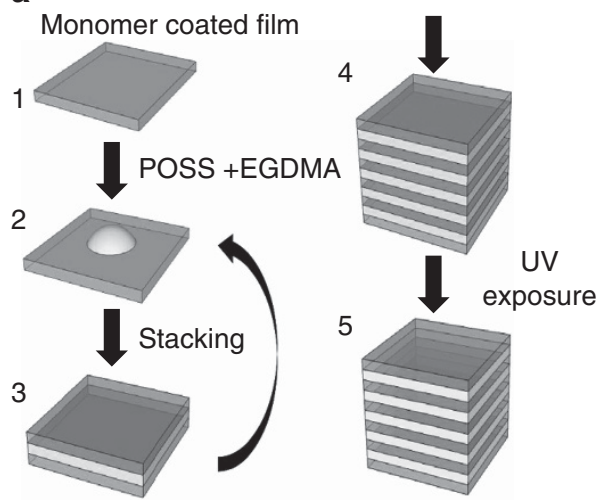

b

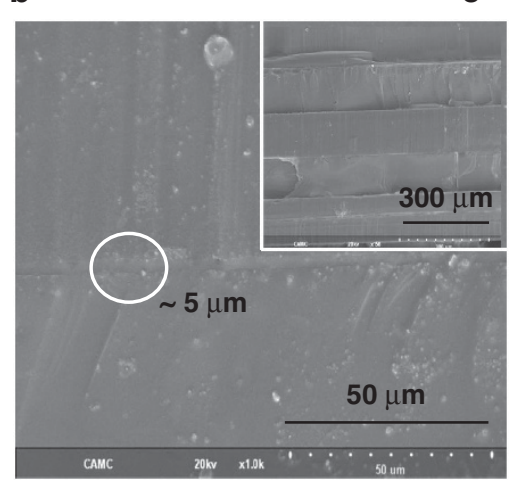

Figure 2 (a) Schematic fabrication process of laminated film composite. The surface-modified PET films developed using the iCVD method are sandwiched with the POSS/EGDMA polymer poured on the film; this is followed by a continuous stacking process to form the laminated film composite. Finally, the composite is exposed to UV radiation for $20 \mathrm{~min}$ to consolidate the inorganic polymer with strong interlayer adhesion. Cross-sectional scanning electron microscopy images: (b) two PET films with a POSS interlayer and (c) laminated film composite at low magnification. A full color version of this figure is available at Polymer Journal online.

composition of the PET film to be systematically tuned. ${ }^{20}$ Figure 3 shows a schematic of the surface modification process for a bare PET film to enhance the wetting of viscous POSS polymer with strong bonding adhesion. Before fabrication of the laminated film composite, V4D4 monomer-an eight-member siloxane ring with four vinyl groups - was polymerized by the iCVD process to form a thin polymer layer on the primer-treated PET film, as shown in Figure 3a. The vinyl groups of V4D4 monomer reacted with the activated groups on the primer-treated PET film, and the remaining vinyl groups of V4D4 interacted with the acrylate groups of POSS upon layering. This eventually led to enhanced bonding between both the PET substrate and POSS through the formation of a crosslinked three-dimensional network after UV radiation. In addition, we used atomic force microscopy to measure the overall coating
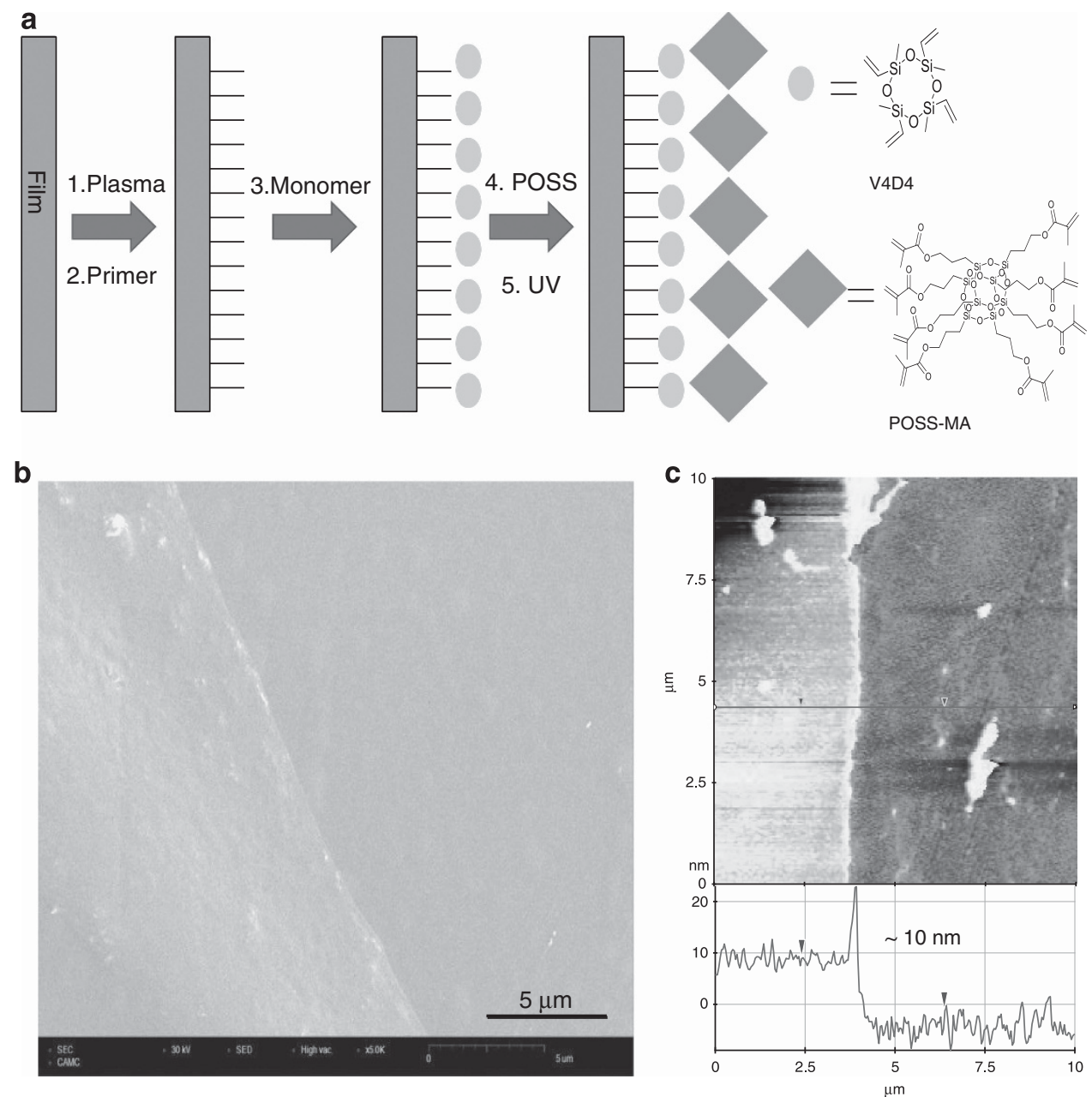

Figure 3 (a) Overall fabrication process from bare PET film to POSS inorganic interlayer. (b) Scanning electron microscopy image of V4D4-coated PET film. The left section is the V4D4-coated PET film. The right section is a bare PET film. (c) Atomic force microscopy image of V4D4 polymer; thickness: 10 nm. A full color version of this figure is available at Polymer Journal online. 


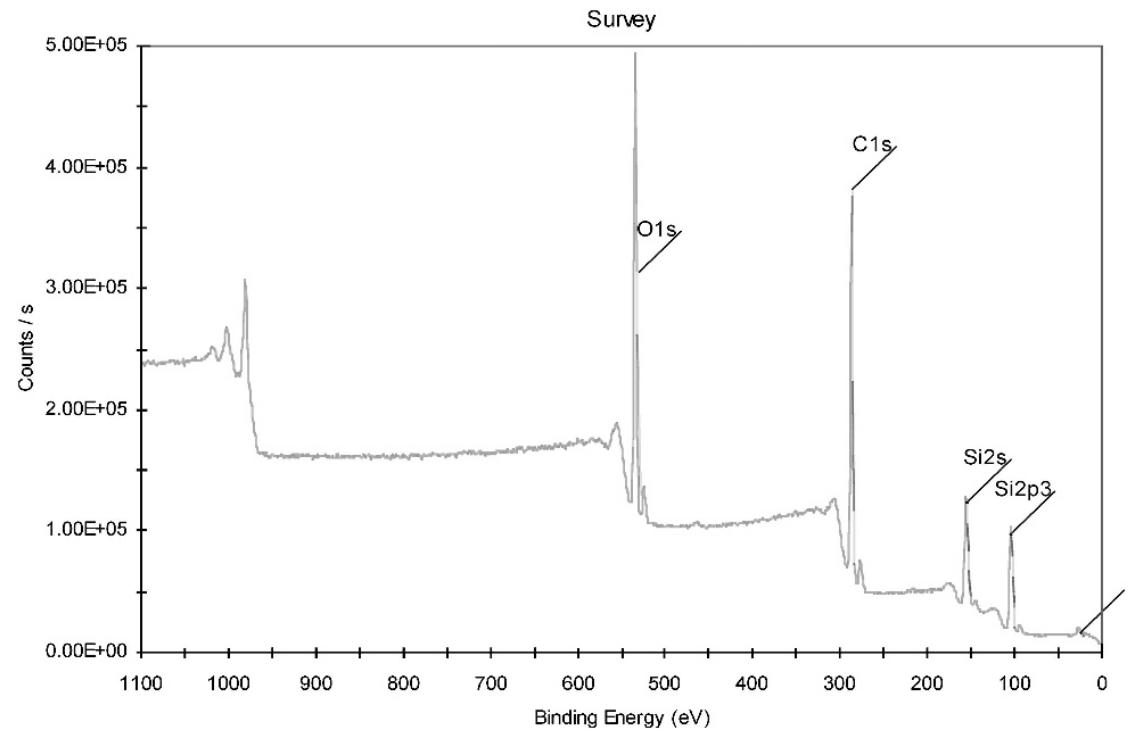

Figure 4 X-ray photoelectron spectroscopy (XPS) survey scans of V4D4 polymer with $10 \mathrm{~nm}$ thickness on PET film. A full color version of this figure is available at Polymer Journal online.

Table 1 Mechanical strength and optical properties of various samples

\begin{tabular}{lccc}
\hline \multirow{2}{*}{ Substrate (depth, $\mu \mathrm{m})$} & \multicolumn{2}{c}{ Optical properties } \\
\cline { 3 - 4 } & $\begin{array}{c}\text { Mechanical property } \\
\text { Young's modulus (GPa) }\end{array}$ & Trans. (\%) & Haze (\%) \\
\hline PET (100) & $2.49 \pm 0.4$ & 91.54 & 4.51 \\
POSS (1000) & $2.07 \pm 0.09$ & 87.9 & 1.3 \\
PET 6 layers + POSS 5 layers (630) & $8.11 \pm 1.47$ & 86.16 & 11.53 \\
Glass (1000) & $36.66 \pm 3.21$ & 91.8 & 0.5 \\
\hline
\end{tabular}

Abbreviations: PET, poly(ethylene terephthalate); POSS, polyhedral oligomeric silsesquioxane.

thickness of the V4D4 polymer on the PET film. The surface interface was used to verify that the polymerized V4D4 layer formed with $\sim 10 \mathrm{~nm}$ thickness.

X-ray photoelectron spectroscopy was employed to elucidate the chemical compositions of the deposited V4D4 polymer on the PET film. Figure 4 shows a survey spectrum of the 10-nm thick V4D4 on the PET film. Successful immobilization of the V4D4 polymer, which comprised a $\mathrm{Si}$ group, was verified by the appearance of the peaks of Si2s $(165 \mathrm{eV})$ and Si2p3 $(104.6 \mathrm{eV})$. The ratio of elemental species was Si:C:O = 22.4:55.37: 22.22 , which was close to the chemical composition of V4D4.

In this work, the thickness of the laminated film composite was controlled to less than $\sim 1 \mathrm{~mm}$ in consideration of its potential for electronic display applications. Six layers of $100-\mu \mathrm{m}$ thick PET film were alternately laminated with five interlayers of 5 - $\mu \mathrm{m}$ thick POSS polymer. Eventually, the transparent mixture of methacrylate-functionalized POSS and EGDMA interlayer was consolidated by UV-initiated radical polymerization throughout the transparent PET film layers to produce rigid and lightweight laminated film composites.

The obtained composite samples were investigated to measure the mechanical and optical properties, which are presented in Table 1. The Young's modulus $E$ of the 11layer laminated composite was measured by the three-point bending test, and those of the POSS and glass were also measured. $E$ was calculated by the following equations:

$$
E_{b}=\frac{P L^{3}}{4 b h^{3} \delta}=\frac{L^{3}}{4 b h^{3}} S
$$

where $L$ is the span length between supports $(\mathrm{m}), b$ is the specimen width $(\mathrm{m}), h$ is the specimen thickness $(\mathrm{m}), P$ is the load increment as measured from the preload $(\mathrm{N}), d$ is the deflection increment at midspan as measured from the preload $(\mathrm{m})$ and $S$ is $P / d$ as a slope. The mechanical properties of the laminated composite gave a Young's modulus $E$ of $8.11 \pm 1.47 \mathrm{GPa}$, which is an obvious improvement in the modulus compared with $2.07 \pm 0.09 \mathrm{GPa}$ of the POSS-only sample.

When the number of POSS layers in the laminated film was increased to six, the light transmittance decreased from $\sim 91.5 \%$ to $\sim 86 \%$ for the PET film; the haze value increased from $\sim 4.5$ to $\sim 11.5$ for the PET film. The results are listed in Table 1. Light must be reflected and scattered at the interface or matrix upon penetration through the laminated film composite, although the refractive indices of 1.57 and 1.46 for PET and POSS, respectively, do not differ greatly. The optical property of the layered film composite was inferior to the transmittance of $91.8 \%$ and haze of 0.5 for glass.

\section{CONCLUSION}

We proposed a new concept and fabrication method for a laminated film composite that uses POSS inorganic interlayers between PET films, and its feasibility as a transparent and hard alternative to glass was tested by selecting commercially available material sources. The POSS/PET film composite was alternately stacked up to 11 layers and then subjected to simple UV curing to obtain consolidated products with $\sim 1 \mathrm{~mm}$ thickness. The laminated film composite showed $22 \%$ of the relative strength compared with original PET film to $6 \%$ of glass and $94 \%$ of the relative transmittance of glass. The mechanical and optical performances of the laminated film composite are not yet satisfactory; however, our results represent a meaningful attempt that demonstrates a new concept of composite fabrication for electronic display applications.

\section{ACKNOWLEDGEMENTS}

This research was supported by grant-in-aid from the Samsung Electronics Co., Ltd, the Creative Research Initiatives project (No. 20120001224) and the University-Institute cooperation program (2102) administered by the Korean Ministry of Education, Science and Technology.

Dong-ki Seo ${ }^{1}$, Jae Bem You ${ }^{2}$, Sung Gap $\mathrm{Im}^{2}$, Jooho Kim ${ }^{3}$, Kyung-kook Kim ${ }^{3}$, Yeon-Kyoung Jung ${ }^{3}$, Ming $\mathrm{Li}^{4}$, Chan Pil Park ${ }^{1}$ and Dong-Pyo Kim ${ }^{5}$

${ }^{1}$ Micro Chemistry Laboratory, Graduate School of Analytical Science \& Technology, Chungnam National University, Daejeon, Korea;

${ }^{2}$ Functional Thin Films Lab, Department of Chemical and Biomolecular Engineering, Korea Advanced Institute of Science and Technology, Daejeon, Korea; ${ }^{3}$ Samsung 
Electronics Co., Ltd, Suwon-Si, Korea; ${ }^{4}$ College of Enviromental Science \& Engineering, Yangzhou University, Yangzhou, China and ${ }^{5}$ Center of Applied Microfluidic Chemistry, Department of Chemical Engineering, Pohang University of Science \&Technology, Pohang, Korea

E-mail: chan@cnu.ac.kr (CPP) E-mail:dpkim@postech.ac.kr (D-PK)

1 Kurkjian, C. R., Kammlott, G. W. \& Chaudhri, M. M. Indentation behavior of soda-lime silica glass, fused silica, and single-crystal quartz at liquid nitrogen temperature. J. Am. Ceram. Soc. 78, 737-744 (1995).

2 Wohlers, H. C., Newstein, H. \& Daunis, D. Carbon Monoxide and sulfur dioxide adsorption on - and desorption from glass, plastic, and metal tubings. J. Air. Pollut. Control Ass. 17, 753-756 (1967).

3 Ottevaere, H., Cox, R., Herzig, H. P., Miyashita, T., Naessens, K., Taghizadeh, M., Völkel, R., Woo, H. J. \& Thienpont, $\mathrm{H}$. Comparing glass and plastic refractive microlenses fabricated with different technologies. J. Opt. A: Pure Appl. Opt. 8, S407 (2006).
4 Norville, H., Harvill, N., Conrath, E., Shariat, S. \& Mallonee, S. Glass-related injuries in Oklahoma city bombing. J. Perform. Constr. Facil. 13, 50-56 (1999).

5 Keeney, A. H., Fintelmann, E. \& Estlow, B. Refractive correction and associated factors in spectacle glass injury. Arch. Ophthalmol. 88, 2-8 (1972).

6 Warburton, A. L. \& Shepherd, J. P. The effectiveness of toughened glass in reducing bar glass injury. J. Dent Res. 79, 515-515 (2000).

7 Glass, C. G. Product information sheet http://www. CorningGorillaGlass.com. (2011)

8 Kistler, S. S. Stresses in glass produced by nonuniform exchange of monovalent ions. J. Am. Ceram. Soc. 45, 59-68 (1962).

9 Acloque, P. \& Tochon, J. Measurement of the mechanical strength of glass after reinforcement. (Comptes Rendus) 687-704 (1962).

10 Thornton, J. I. \& Cashman, P. J. The effect of tempered glass on bullet trajectory. Forensic Sci. 31, 743-746 (1986).

11 Choi, M.-C., Kim, Y. \& Ha, C.-S. Polymers for flexible displays: from material selection to device applications. Prog. Polym. Sci. 33, 581-630 (2008).

12 Shi, M. K., Graff, G. L., Gross, M. E. \& Martin, P. M. In situ and real-time monitoring of plasma-induced etching of PET and acrylic films. Plasmas Polym. 4, 247-258 (1999).

13 Lee, S. H., Kim, H. G. \& Kim, S. S. Organic-inorganic hard coating layer formation on plastic substrate by UV curing process. Macromol. Res. 18, 40-46 (2010).
14 Kim, J. K., Yoon, K. H., Bang, D. S., Park, Y.-B., Kim, H.-U. \& Bang, Y.-H. Morphology and rheological behaviors of poly(ethylene terephthalate) nanocomposites containing polyhedral oligomeric silsesquioxanes. J. Appl. Polym. Sci. 107, 272-279 (2008).

15 Ayandele, E., Sarkar, B. \& Alexandridis, P. Polyhedral oligomeric silsesquioxane (POSS)-containing polymer nanocomposites. Nanomaterials 2, 445-475 (2012).

16 Lichtenhan, J. D., Otonari, Y. A. \& Carr, M. J. Linear hybrid polymer building blocks: methacrylate-functionalized polyhedral oligomeric silsesquioxane monomers and polymers. Macromolecules 28, 8435-8437 (1995).

17 Siracusa, V., Rocculi, P., Romani, S. \& Rosa, M. D. Biodegradable polymers for food packaging: a review. Trends Food Sci. Technol. 19, 634-643 (2008).

18 Trujillo, N. J., Wu, Q. \& Gleason, K. K. Ultralow dielectric constant tetravinyltetramethylcyclotetrasiloxane films deposited by initiated chemical vapor deposition (iCVD). Adv. Funct. Mater. 20, 607-616 (2010).

19 Pielichowski, K., Njuguna, J., Janowski, B. \& Pielichowski, J. Polyhedral oligomeric silsesquioxanes (POSS)-containing nanohybrid polymers. Adv. Polym. Sci. 201, 225-296 (2006).

20 Baxamusa, S. H., Im, S. G. \& Gleason, K. K. Initiated and oxidative chemical vapor deposition: a scalable method for conformal and functional polymer films on real substrates. Phys. Chem. Chem. Phys. 11, 5227-5240 (2009). 